\title{
Reviewing the manifold aspects Ganciclovir crystal forms
}

José A. Fernandes, Simona Galli, ${ }^{*}$ Giovanni Palmisano, Paolo Volante,

Ricardo F. Mendes, Filipe A. Almeida Paz and Norberto Masciocchi

ELECTRONIC SUPPLEMENTARY INFORMATION 

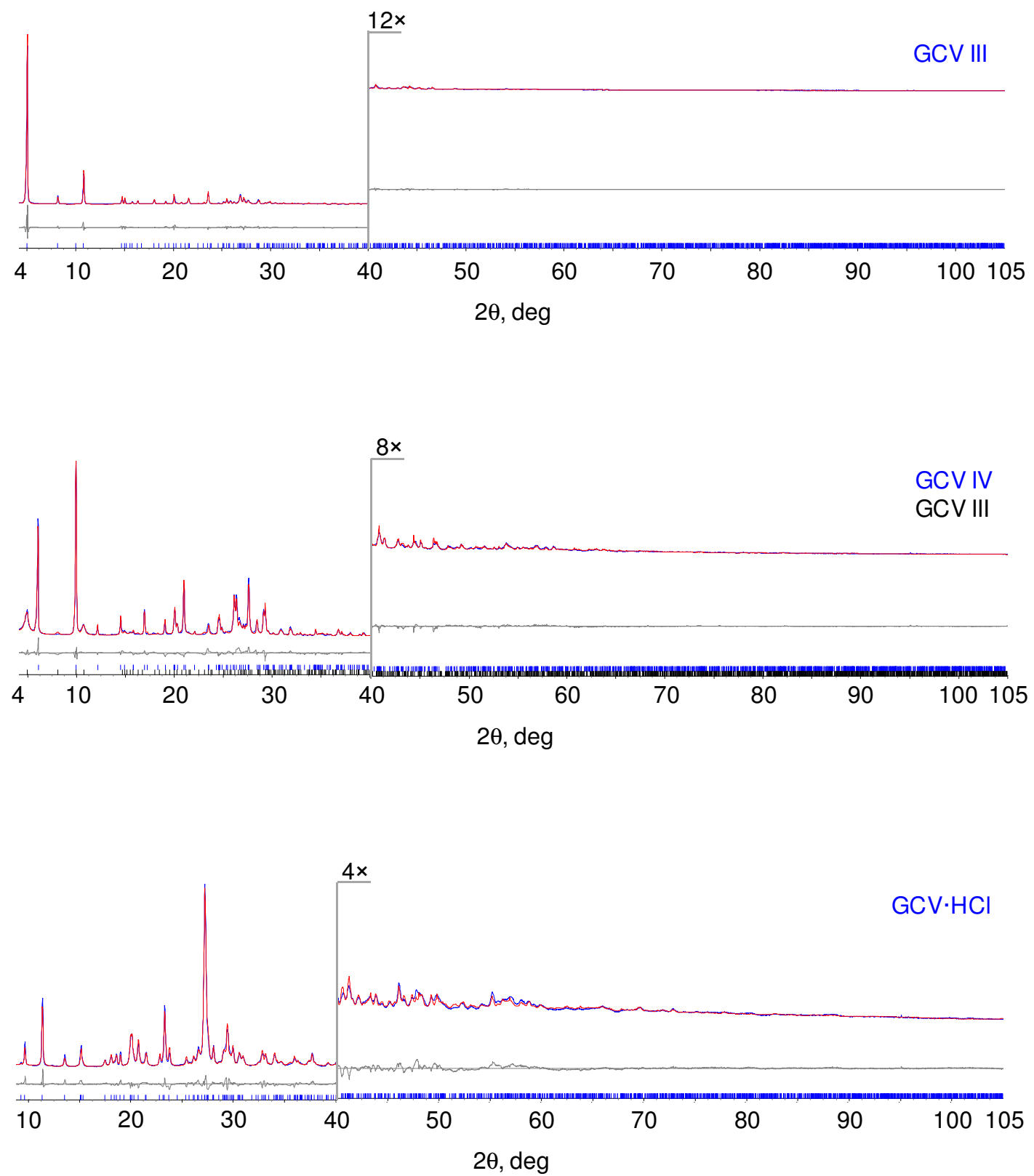

Figure S1. From top to bottom: graphical results of the final Rietveld refinements carried out on the PXRD data of form III, form IV (impure of III) and GCV $\cdot \mathbf{H C l}$, in terms of experimental, calculated and difference traces (blue, red and gray, respectively). The blue markers at the bottom indicate the position of the Bragg's reflections. Horizontal axis, $2 \theta$ (deg); vertical axis, intensity (counts). The portion above $40^{\circ}(2 \theta)$ has been magnified. 

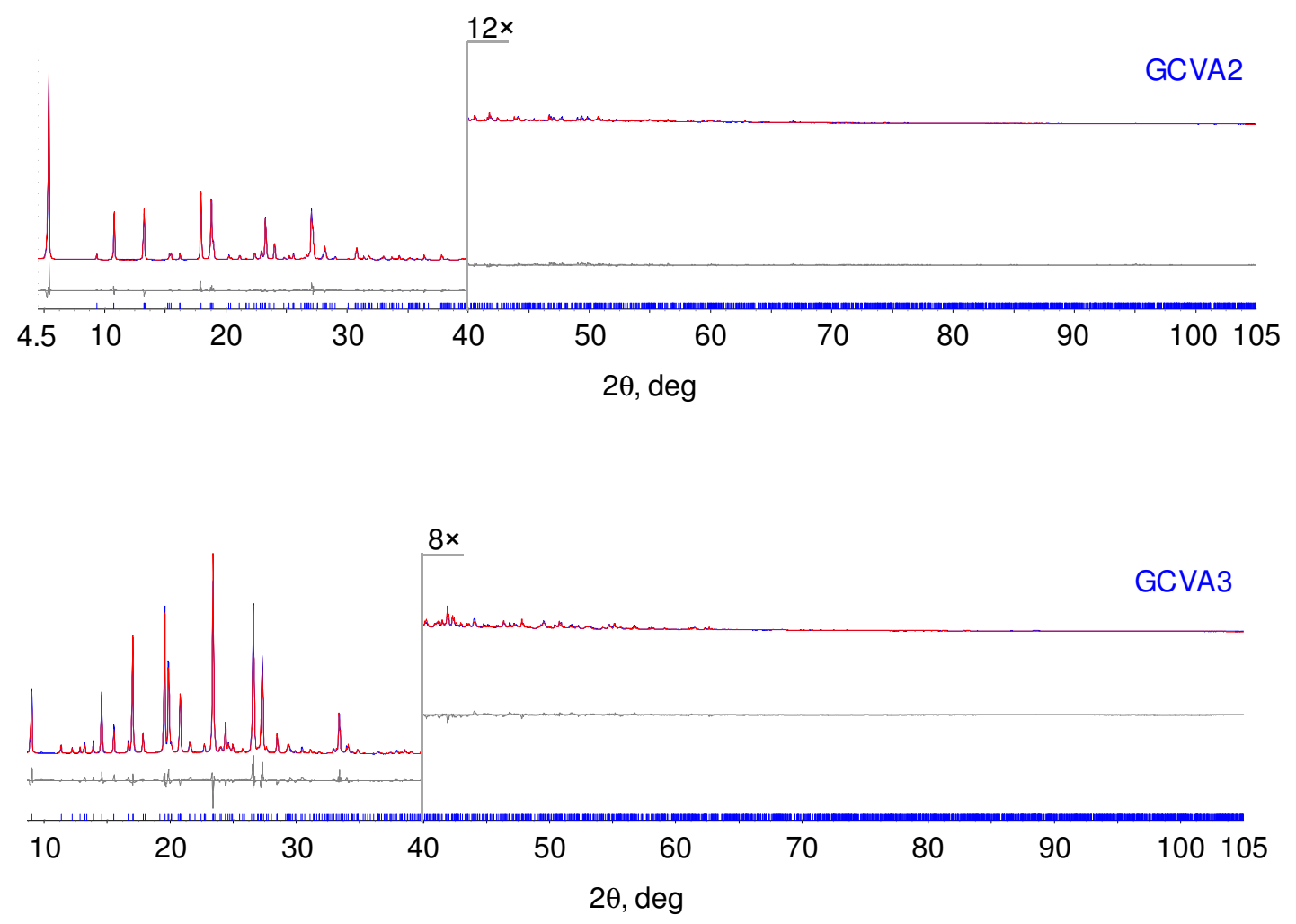

Figure S2. From top to bottom: graphical results of the final Rietveld refinements carried out on the PXRD data of GCVA2 and GCVA3, in terms of experimental, calculated and difference traces (blue, red and gray, respectively). The blue markers at the bottom indicate the position of the Bragg's reflections. Horizontal axis, $2 \theta$ (deg); vertical axis, intensity (counts). The portion above $40^{\circ} 2 \theta$ has been magnified. 


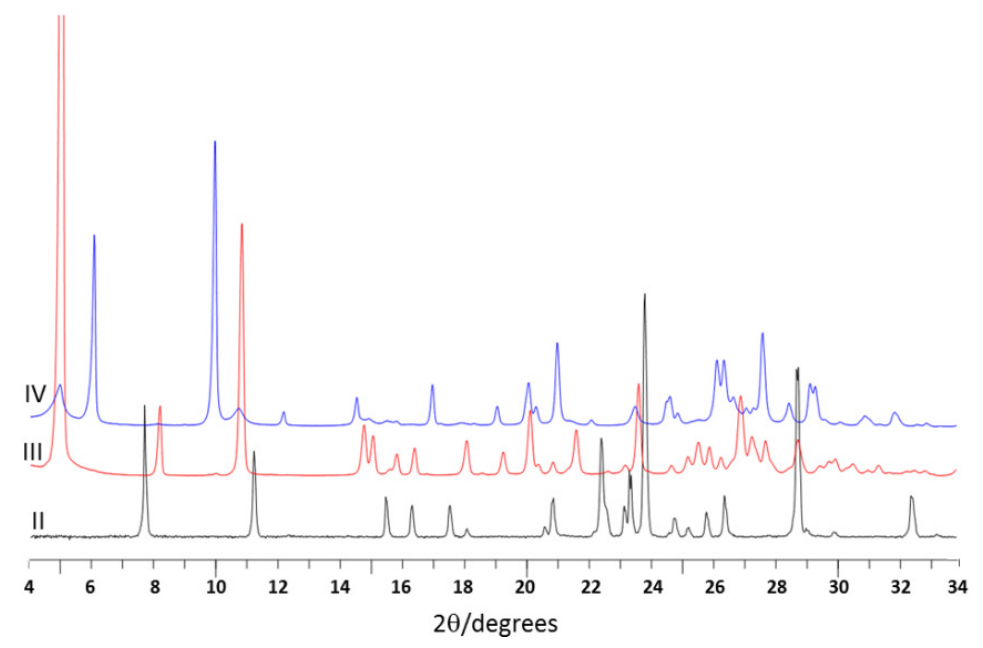

Figure S3. Low-to medium angle portion of the experimental PXRD patterns of forms II, III and IV.

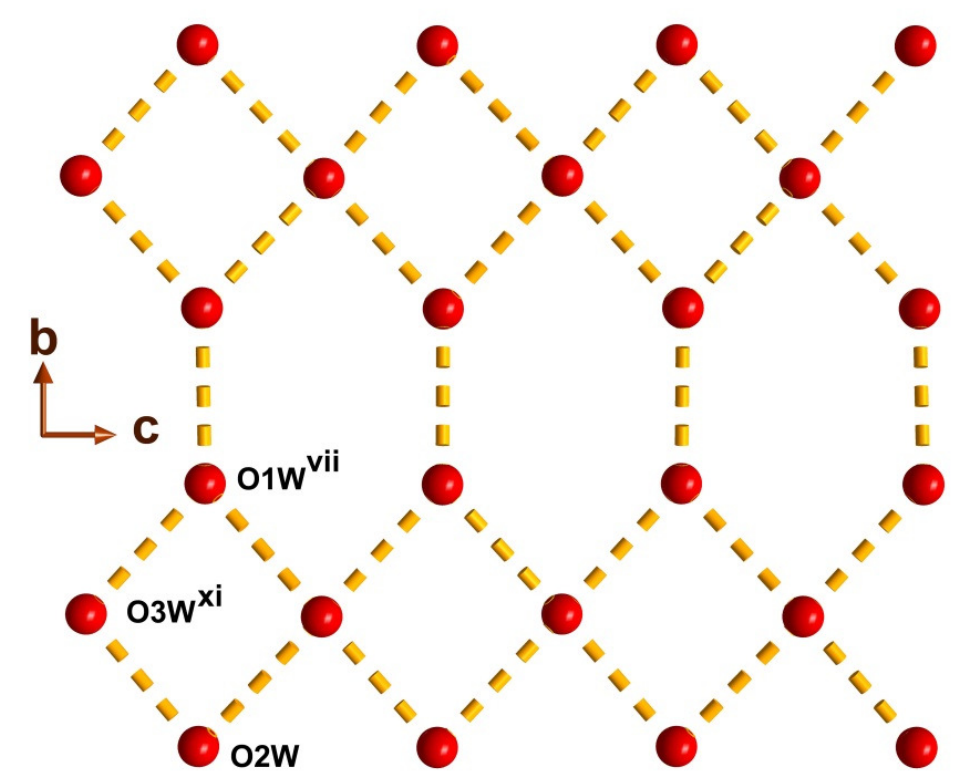

Figure S4. The quasi planar network formed by the water molecules present in the 1-D channels of form III, as retrieved by powder X-ray diffraction. The hydrogen bond interactions are represented with yellow dashed lines $(\mathrm{O} \cdots \mathrm{O} 2.54(2)-2.76(3) \AA$ ). Symmetry codes: (vii) $x, 1 / 2-y$, $1 / 2+z ;(x i) x, y, 1+z$. See Table S3 for details on hydrogen bond distances and angles. 

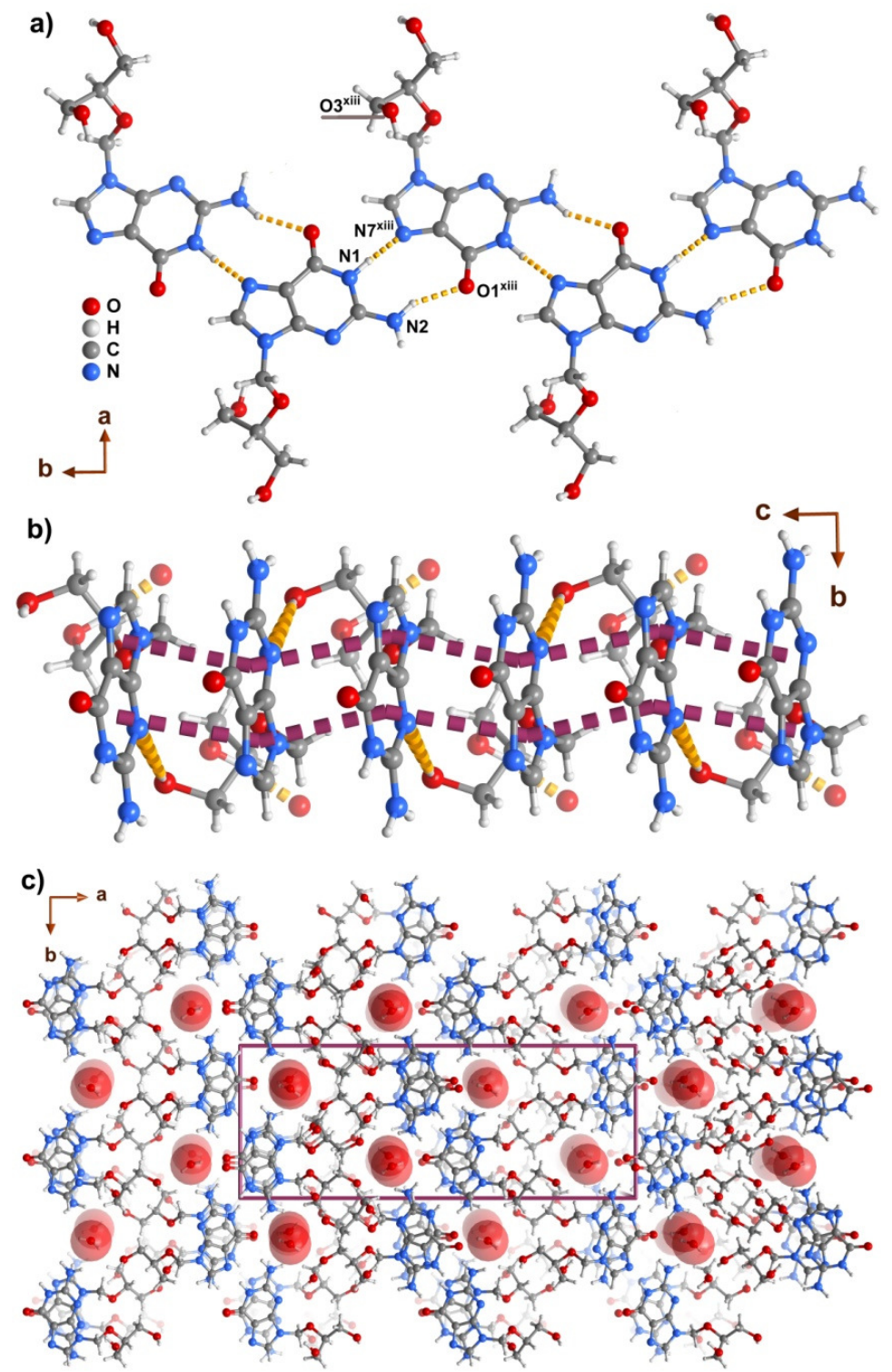

Figure S5. Representation of the crystal structure of form IV. a) Portion of the 1-D hydrogenbonded chain of guanine moieties running along the [010] direction. The hydrogen bonds have been depicted with yellow dashed lines. b) The $\pi \cdots \pi$ stacking taking place along the [001] direction between nearby guanine moieties. The violet dashed lines have been added to guide the eye. c) The packing, viewed in perspective along $c$ : the 1-D channels hosting water molecules are clearly smaller than those present in form III (Figure 2c). See Tables S4 and S8 for details on the supramolecular bond distances and angles. Symmetry code: (xiii) $2-x, 1 / 2+y, 1 / 2-z$. 


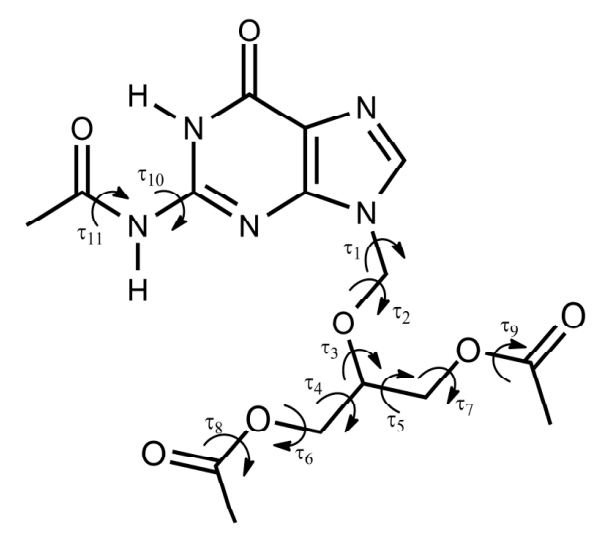

Chart S1. Molecular structure of GCVA3 showing the torsion angles optimized during the structural determination/refinement of III, IV, GCV·HCl, GCVA2 and GCVA3. $\tau_{1}=\mathrm{O}_{2}-\mathrm{C}_{10^{-}}$ $\mathrm{N}_{9}-\mathrm{C}_{4} ; \tau_{2}=\mathrm{C}_{11}-\mathrm{O}_{2}-\mathrm{C}_{10}-\mathrm{N}_{9} ; \tau_{3}=\mathrm{C}_{12}-\mathrm{C}_{11}-\mathrm{O}_{2}-\mathrm{C}_{10} ; \tau_{4}=\mathrm{O}_{4}-\mathrm{C}_{13}-\mathrm{C}_{11}-\mathrm{O}_{2} ; \tau_{5}=\mathrm{O}_{4}-\mathrm{C}_{12}-\mathrm{C}_{11}-\mathrm{O}_{2} ; \tau_{6}=$ $\mathrm{C}_{16}-\mathrm{O}_{4}-\mathrm{C}_{13}-\mathrm{O}_{11} ; \tau_{7}=\mathrm{C}_{14}-\mathrm{O}_{3}-\mathrm{C}_{12}-\mathrm{C}_{11} ; \tau_{8}=\mathrm{C}_{17}-\mathrm{C}_{16}-\mathrm{O}_{4}-\mathrm{C}_{13} ; \tau_{9}=\mathrm{C}_{15}-\mathrm{C}_{14}-\mathrm{O}_{3}-\mathrm{C}_{12}$

Table S1. The actual values (in degrees, e.s.d.s in parentheses) adopted by the torsion angles $\tau_{1}-\tau_{9}$ (see also Chart S1) in III, IV, GCV·HCI, GCVA2 and GCVA3.

\begin{tabular}{|l|r|r|r|r|r|r|r|r|r|}
\hline Phase & \multicolumn{1}{|c|}{$\boldsymbol{\tau}_{\mathbf{1}}$} & \multicolumn{1}{c|}{$\boldsymbol{\tau}_{\mathbf{2}}$} & \multicolumn{1}{c|}{$\boldsymbol{\tau}_{\mathbf{3}}$} & \multicolumn{1}{c|}{$\boldsymbol{\tau}_{\mathbf{4}}$} & \multicolumn{1}{c|}{$\boldsymbol{\tau}_{\mathbf{5}}$} & $\boldsymbol{\tau}_{\mathbf{6}}$ & $\boldsymbol{\tau}_{\mathbf{7}}$ & $\boldsymbol{\tau}_{8}$ & $\boldsymbol{\tau}_{9}$ \\
\hline III & $76.7(4)$ & $115.6(5)$ & $88.8(4)$ & $84(2)$ & $\begin{array}{r}-75(2) \\
88(2)\end{array}$ & & & & \\
\hline IV & $59.5(3)$ & $127.4(4)$ & $-70.9(4)$ & $-166.0(8)$ & $-82(1)$ & & & & \\
\hline G·HCl & $100.7(2)$ & $-95.2(3)$ & $-75.8(3)$ & $-59.1(8)$ & $-46.85(7)$ & & & & \\
\hline GA2 & $119.9(2)$ & $-72.3(2)$ & $168.0(2)$ & $-74.7(6)$ & $-55.4(4)$ & $176.6(6)$ & $-76.0(4)$ & $-69.0(8)$ & $175 .(6)$ \\
\hline GA3 & $56.0(2)$ & $79.7(2)$ & $83.45(2)$ & $-157.0(6)$ & $68.8(4)$ & $-105.4(6)$ & $-163.0(4)$ & $172.6(8)$ & $170.7(4)$ \\
\hline$\tau_{1}=\mathrm{O}_{2}-\mathrm{C}_{10}-\mathrm{N}_{9}-\mathrm{C}_{4} ; \tau_{2}=\mathrm{C}_{11}-\mathrm{O}_{2}-\mathrm{C}_{10}-\mathrm{N}_{9} ; \tau_{3}=\mathrm{C}_{12}-\mathrm{C}_{11}-\mathrm{O}_{2}-\mathrm{C}_{10} ; \tau_{4}=\mathrm{O}_{4}-\mathrm{C}_{13}-\mathrm{C}_{11}-\mathrm{O}_{2} ; \tau_{5}=\mathrm{O}_{4}-\mathrm{C}_{12}-\mathrm{C}_{11}-\mathrm{O}_{2} ;$ \\
$\tau_{6}=\mathrm{C}_{16}-\mathrm{O}_{4}-\mathrm{C}_{13}-\mathrm{O}_{11} ; \tau_{7}=\mathrm{C}_{14}-\mathrm{O}_{3}-\mathrm{C}_{12}-\mathrm{C}_{11} ; \tau_{8}=\mathrm{C}_{17}-\mathrm{C}_{16}-\mathrm{O}_{4}-\mathrm{C}_{13} ; \tau_{9}=\mathrm{C}_{15}-\mathrm{C}_{14}-\mathrm{O}_{3}-\mathrm{C}_{12}$. \\
\hline
\end{tabular}


Table S2. Selected hydrogen bond distances and angles for GCV form $\mathbf{I I}^{a, b}$

\begin{tabular}{|l|r|r|}
\hline $\mathbf{D}-\mathbf{H} \cdots \mathbf{A}$ & $\mathbf{D} \cdots \mathbf{A}, \mathbf{A}$ & $\mathbf{D}-\mathbf{H} \cdots \mathbf{A}, \mathbf{d e g}$ \\
\hline $\mathrm{N} 1-\mathrm{H} 1 \mathrm{X} \cdots \mathrm{N} 7^{i}$ & $2.841(12)$ & 166 \\
\hline $\mathrm{N} 2-\mathrm{H} 2 \mathrm{X} \cdots \mathrm{O} 4^{i i}$ & $3.085(11)$ & 141 \\
\hline $\mathrm{N} 2-\mathrm{H} 2 \mathrm{Y} \cdots \mathrm{O} 1^{i}$ & $2.848(11)$ & 162 \\
\hline $\mathrm{O} 3-\mathrm{H} 3 Z \cdots \mathrm{N} 3$ & $3.008(12)$ & 136 \\
\hline $\mathrm{O} 4-\mathrm{H} 4 Z \cdots \mathrm{O} 1^{i i i}$ & $2.871(9)$ & 151 \\
\hline $\begin{array}{l}\text { a) For comparison purposes. The labels of the atoms } \\
\text { reported in Ref. 1 have been changed in order to allow } \\
\text { direct comparison with the results presented in this } \\
\text { work. } b) \text { Symmetry codes: (i) }-x,-1 / 2+y,-z ;(i i) 2-x,-1 / 2+y, \\
1-z ; \text { (iii) } 1+x, y, 1+z .\end{array}$
\end{tabular}

Table S3. Selected hydrogen bond distances and angles for GCV form III. ${ }^{a}$

\begin{tabular}{|c|c|c|}
\hline $\mathbf{D}-\mathbf{H} \cdots \mathbf{A}$ & $\mathbf{D} \cdots \mathbf{A}, \AA$ & D-H $\cdots A$, deg \\
\hline $\mathrm{N} 1-\mathrm{H} 1 \mathrm{X} \cdots \mathrm{N} 7^{i v}$ & $2.78(3)$ & 171 \\
\hline $\mathrm{N} 2-\mathrm{H} 2 \mathrm{X} \cdots \mathrm{O} 2 \mathrm{~W}^{v}$ & 2.93(4) & 124 \\
\hline $\mathrm{N} 2-\mathrm{H} 2 \mathrm{X}^{\cdots} \cdot \mathrm{O} 4^{v i}$ & $3.05(3)$ & 140 \\
\hline $\mathrm{N} 2-\mathrm{H} 2 \mathrm{Y}^{\cdots} \mathrm{O} 1^{i v}$ & $3.14(3)$ & 160 \\
\hline $\mathrm{O} 3 \mathrm{P}-\mathrm{H} 3 \mathrm{P} \cdots \mathrm{O} 1 \mathrm{~W}^{v i i}$ & $2.64(2)$ & 163 \\
\hline $\mathrm{O} 4-\mathrm{H} 4 \mathrm{Z} \cdots \mathrm{N} 3^{\text {viii }}$ & $2.87(3)$ & 135 \\
\hline $\mathrm{O} 1 \mathrm{~W} \cdots \mathrm{O} 1 \mathrm{~W}^{i x}$ & $2.55(1)$ & \\
\hline $\mathrm{O} 1 \mathrm{~W} \cdots \mathrm{O} 3 \mathrm{~W}^{x}$ & $2.66(2)$ & \\
\hline $\mathrm{O} 1 \mathrm{~W} \cdots \mathrm{O} 3 \mathrm{~W}^{v i i}$ & $2.54(2)$ & \\
\hline $\mathrm{O} 2 \mathrm{~W} \cdots \mathrm{O} 3 \mathrm{~W}^{x i}$ & $2.53(2)$ & \\
\hline $\mathrm{O} 2 \mathrm{~W} \cdots \mathrm{O} 3 \mathrm{~W}^{x i i}$ & $2.76(3)$ & \\
\hline \multicolumn{3}{|c|}{ (a) Symmetry codes: (iv) $\frac{1}{1 / 2+x}, 1-y, 1.5-z ;(v) 1+x, y, z ;(v i)$} \\
\hline \multicolumn{3}{|c|}{$\begin{array}{l}1.5-x, y,-1 / 2+z ;(\text { vii) } x, 1 / 2-y, 1 / 2+z ; \text { (viii) } 1.5-x, y, 1 / 2+z ; \text { (ix) })^{1 / 2-} \\
x, 1 / 2-y, z ;(x)^{1 / 2-x}, 1 / 2-y, 1+z ;(x i) x, y, 1+z ;(x i i) 1 / 2-x, y, 1.5+z .\end{array}$} \\
\hline
\end{tabular}

(1) Kawamura, T.; Hirayama, N. X-ray Struct. Anal. Online 2009, 25, 51-52. 
Table S4. Selected hydrogen bond distances and angles for GCV form IV. ${ }^{a}$

\begin{tabular}{|c|c|c|}
\hline $\mathrm{D}-\mathrm{H} \cdots \mathbf{A}$ & $\mathbf{D} \cdots \mathbf{A}, \AA$ & D-H $\cdots A$, deg \\
\hline $\mathrm{N} 1-\mathrm{H} 1 \mathrm{X} \cdots \mathrm{N} 7^{x i i i}$ & $2.756(7)$ & 168 \\
\hline $\mathrm{N} 2-\mathrm{H} 2 \mathrm{Y}^{\cdots} \cdots \mathrm{O} 1^{x i i i}$ & $3.040(8)$ & 157 \\
\hline $\mathrm{O} 3-\mathrm{H} 3 \mathrm{Z} \cdots \mathrm{N} 3^{v i i}$ & $3.066(6)$ & 154 \\
\hline $\mathrm{O} 4-\mathrm{H} 4 \mathrm{Z} \cdots \mathrm{O} 3^{x i v}$ & $2.904(8)$ & 172 \\
\hline $\mathrm{O} 1 \mathrm{~W}-\mathrm{H} 1 \mathrm{~W} \cdots \mathrm{O} 1^{x v}$ & $3.07(10)$ & 171 \\
\hline $\mathrm{O} 1 \mathrm{~W}-\mathrm{H} 2 \mathrm{~W} \cdots \mathrm{O} 4^{x v i}$ & $2.96(10)$ & 153 \\
\hline
\end{tabular}

Table S5. Selected hydrogen bond distances and angles for GCV·HCl. ${ }^{a}$

\begin{tabular}{|c|c|c|}
\hline $\mathrm{D}-\mathbf{H} \cdots \mathbf{A}$ & $\mathbf{D} \cdots \mathbf{A}, \AA$ & D-H $\cdots A$, deg \\
\hline $\mathrm{N} 1-\mathrm{H} 1 \mathrm{X} \cdots \mathrm{O} 4^{x v i i}$ & $2.830(8)$ & 158 \\
\hline $\mathrm{N} 2-\mathrm{H} 2 \mathrm{X}^{\cdots} \cdot \mathrm{O} 3^{x v i i i}$ & $3.092(10)$ & 159 \\
\hline $\mathrm{N} 2-\mathrm{H} 2 \mathrm{Y} \cdots \mathrm{Cl1}{ }^{x i x}$ & $3.321(8)$ & 153 \\
\hline $\mathrm{N} 7-\mathrm{H} 7 \mathrm{X} \cdots \mathrm{Cl} 1^{x x}$ & $3.104(10)$ & 176 \\
\hline $\mathrm{O} 3-\mathrm{H} 3 \mathrm{Z} \cdots \mathrm{Cl1}^{x x i}$ & $3.115(7)$ & 105 \\
\hline $\mathrm{O} 3-\mathrm{H} 3 \mathrm{Z} \cdots \mathrm{N} 2^{x v i i i}$ & $3.092(10)$ & 111 \\
\hline $\mathrm{O} 4-\mathrm{H} 4 \mathrm{Z} \cdots \mathrm{Cl1}{ }^{x x i i}$ & $3.038(9)$ & 126 \\
\hline \multicolumn{3}{|c|}{$\begin{array}{l}\text { (a) Symmetry codes: (xvii) }-x, 2-y,-z ;(x v i i i)-x, 3-y,-z ; \\
\text { (xix) } x, 1+y, z ;(x x) x, y,-1+z ;(x x i) x, y,-1+z ;(x x i i)-x, 1-y \text {, } \\
-z \text {. }\end{array}$} \\
\hline
\end{tabular}


Table S6. Selected hydrogen bond distances and angles for GCVA2. ${ }^{a}$

\begin{tabular}{|l|r|r|}
\hline $\mathbf{D}-\mathbf{H} \cdots \mathbf{A}$ & $\mathbf{D} \cdots \mathbf{A}, \mathbf{A}$ & $\mathbf{D}-\mathbf{H} \cdots \mathbf{A}, \mathbf{d e g}$ \\
\hline $\mathrm{N} 1-\mathrm{H} 1 \mathrm{X} \cdots \mathrm{N}^{x x i i i}$ & $2.967(8)$ & 170 \\
\hline $\mathrm{N} 2-\mathrm{H} 2 \mathrm{X} \cdots \mathrm{O}^{x x i v}$ & $3.012(5)$ & 153 \\
\hline $\mathrm{N} 2-\mathrm{H} 2 \mathrm{Y} \cdots \mathrm{O}^{\text {xxiii }}$ & $2.893(9)$ & 146 \\
\hline $\mathrm{C} 12-\mathrm{H} 12 \mathrm{~A} \cdots \mathrm{O}^{x x v}$ & $3.311(8)$ & 141 \\
\hline $\mathrm{C} 17^{x x v}-\mathrm{H} 17 \mathrm{C}^{x x v} \cdots \mathrm{O} 3$ & $3.456(9)$ & $157^{\circ}$ \\
\hline $\begin{array}{l}\text { (a) Symmetry codes: }(x x i i i) \\
(x x v) 2-x, 1 / 2+y, 1 / 2-z ;(x x i v) x, 1 / 2-y,-1 / 2+z\end{array}$ \\
\hline
\end{tabular}

Table S7. Selected hydrogen bond distances and angles for GCVA3. ${ }^{a}$

\begin{tabular}{|l|r|r|}
\hline $\mathbf{D}-\mathbf{H} \cdots \mathbf{A}$ & $\mathbf{D} \cdots \mathbf{A}, \mathbf{A}$ & $\mathbf{D}-\mathbf{H} \cdots \mathbf{A}, \mathbf{d e g}$ \\
\hline $\mathrm{N} 1-\mathrm{H} 1 \mathrm{X} \cdots \mathrm{O} 7$ & $2.556(4)$ & 130 \\
\hline $\mathrm{N} 2^{x x v i}-\mathrm{H} 2 \mathrm{X} \cdots \mathrm{O} 7$ & $2.898(7)$ & 165 \\
\hline $\mathrm{C} 11-\mathrm{H} 11 \cdots \mathrm{O} 5^{x x v i i}$ & $3.281(2)$ & 131 \\
\hline $\begin{array}{l}\text { (a) Symmetry code: }(x x v i) \\
1 / 2+z .\end{array}$ \\
\hline
\end{tabular}

Table S8. $\pi \cdots \pi$ interactions: distance between centroids. ${ }^{a, b}$

\begin{tabular}{|l|l|r|}
\hline Compound & $\mathbf{C g} \cdots \mathbf{C g}$ & Distance, $\AA$ \\
\hline \multirow{2}{*}{ GCV form III } & $\mathrm{Cg} 1 \cdots \mathrm{Cg} 2^{v i}$ & $3.42(3)$ \\
\cline { 2 - 3 } & $\mathrm{Cg} 1 \cdots \mathrm{Cg} 2^{\text {viii }}$ & $3.66(3)$ \\
\hline \multirow{2}{*}{ GCV form IV } & $\mathrm{Cg} 1 \cdots \mathrm{Cg} 2^{x x i v}$ & $3.508(3)$ \\
\cline { 2 - 3 } & $\mathrm{Cg} 1 \cdots \mathrm{Cg} 2^{\text {vii }}$ & $3.883(3)$ \\
\hline \multirow{2}{*}{ GCV·HCl } & $\mathrm{Cg} 2 \cdots \mathrm{Cg} 2^{x v i i}$ & $3.573(3)$ \\
\hline $\begin{array}{l}\text { (a) } \mathrm{Cg} 1: \text { centroid of }\{\mathrm{C} 4, \mathrm{C} 5, \mathrm{~N} 7, \mathrm{C} 8, \mathrm{~N} 9\} ; \mathrm{Cg} 2: \text { centroid } \\
\text { of }\{\mathrm{N} 1, \mathrm{C} 2, \mathrm{~N} 3, \mathrm{C} 4, \mathrm{C} 5, \mathrm{C} 6\} .(b) \text { Symmetry codes: (vi) }\end{array}$ \\
$\begin{array}{l}\text { 1.5- } x, y,-1 / 2+z ;(\text { vii }) x, 1 / 2-y, 1 / 2+z ;(\text { viii }) \\
(x v i .5-x, y, 1 / 2+z ;\end{array}$ \\
\hline
\end{tabular}



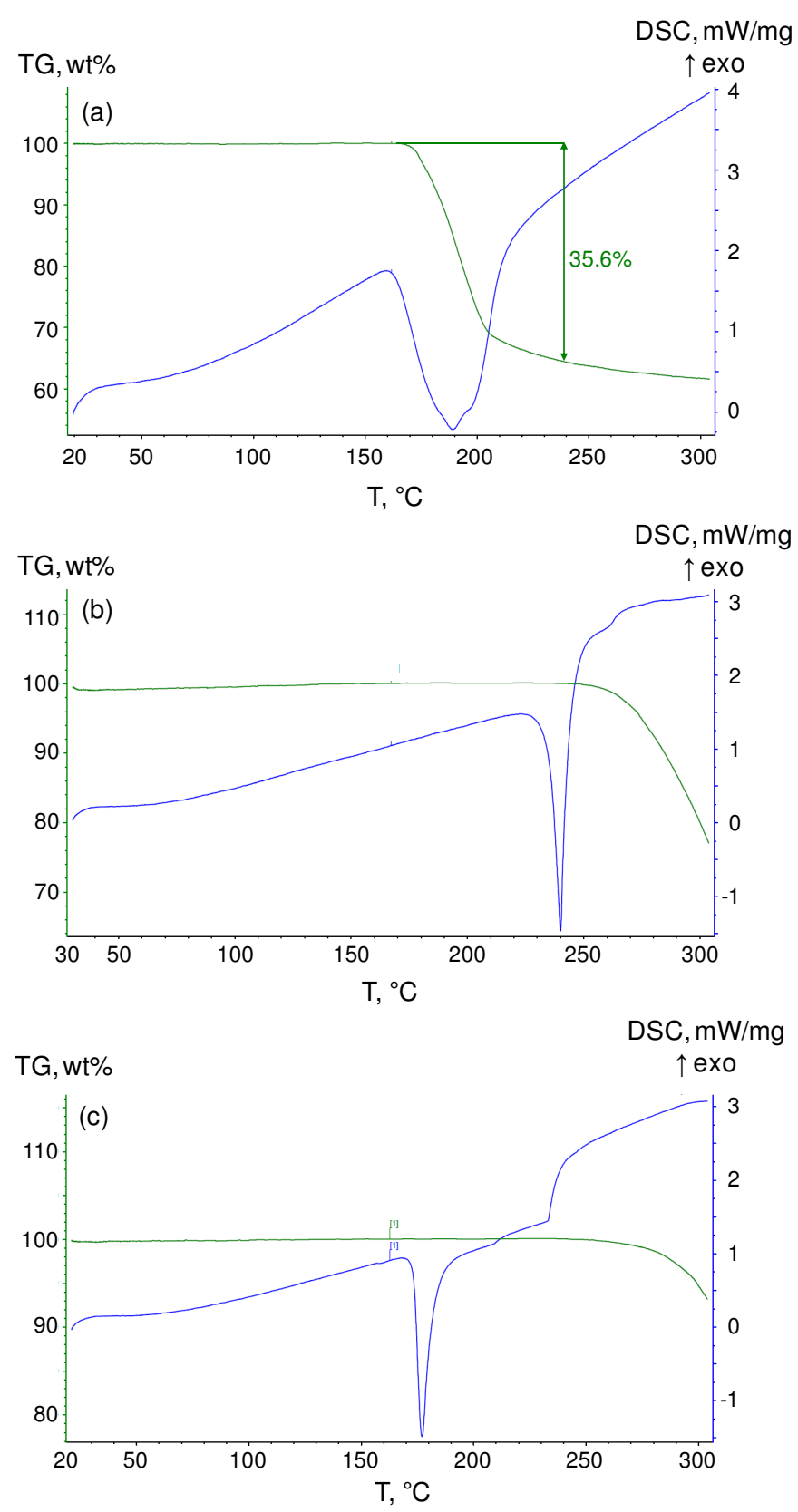

Figure S6. TGA (green) and DSC traces of: (a) GCV·HCl, (b) GCVA2, (c) GCVA3. In (a), the broad endothermic peak centered at $190{ }^{\circ} \mathrm{C}$ is due to the overlapping of melting and decomposition. In (b), the sharp endothermic peak centered at $240{ }^{\circ} \mathrm{C}$ is due to melting of GCVA2, followed by decomposition, starting at $250{ }^{\circ} \mathrm{C}$. In (c), the sharp endothermic peak centered at $177^{\circ} \mathrm{C}$ is due to melting of GCVA3. Decomposition begins only at $250{ }^{\circ} \mathrm{C}$. 